\title{
FRW and Bianchi type I cosmology of f-essence
}

\author{
M. Jamil ${ }^{1} \bullet$ D. Momeni ${ }^{2} \bullet$ N.S. Serikbayev ${ }^{3} \bullet$ \\ R. Myrzakulov ${ }^{3}$
}

\begin{abstract}
F-essence is a generalization of the usual Dirac model with the nonstandard kinetic term. In this paper, we introduce a new model of spinor cosmology containing both Ricci scalar and the non minimally coupled spinor fields in its action. We have investigated the cosmology with both isotropy and anisotropy, where the equations of motion of FRW and Bianchi type-I spacetimes have been derived and solved numerically. Finally the quantization of these models through Wheeler-De Witt (WD) wave function has been discussed.
\end{abstract}

Keywords Spinor Cosmology, Spinor Fields, FRW Model, Bianchi Type I Model

\section{Introduction}

Astrophysical data of supernovae of type Ia indicate that we live in an accelerated expansion era of the Universe (Perlmutter S. 1999; Riess A.G. et al. 1998). There are two major theoretical explanations for this phenomena: the first category is the fluid model in

\section{Jamil}

Center for Advanced Mathematics and Physics, National University of Sciences and Technology, Islamabad, Pakistan

\section{Momeni}

Department of Physics, Faculty of sciences, Tarbiat Moa'llem university, Tehran, Iran

N.S. Serikbayev

Eurasian International Center for Theoretical Physics, Eurasian National University, Astana, Kazakhstan

R. Myrzakulov

Department of Physics, California State University, Fresno, CA 93740 USA

${ }^{1}$ mjamil@camp.nust.edu.pk

22d.momeni@tmu.ac.ir; d.momeni@yahoo.com

33rmyrzakulov@csufresno.edu; rmyrzakulov@gmail.com which we keep the Einstein gravity as a dominant theory and introduce a fluid in the right hand side of the Einstein field equation and then investigate the cosmological evolution of the model (Copeland E. J. et al. 2006) and Amendola L. 2000, paper II). Another approach which has been investigated in recent years is the geometrical one in which we search for generalized models of the gravity which can deduce the accelerated expansion of the Universe Elizalde E. et al. 2004, paper I). Some examples of later model is $f(R)$ gravity (Nojiri S. et al. 2003,2007,2011; Cognola G. et al. 2008; Azadi M. et al. 2008), (Elizalde E. et al. 2004, paper III), (Jamil M. et al. 2011, paper I) and (Momeni D. et al. 2009, paper I) in which the only dynamical sector of action is a function of the Ricci scalar. Here the nonlinear terms of the curvature can be regarded as an alternative for the accelerated expansion of the Universe. Another class of models are the $f(R, G)$ models, where both curvature and Gauss-Bonnet terms as the dynamical quantities (Elizalde E. et al. 2004, paper II), (Bamba K. et al. 2010)and (Felice A. et al. 2010). Another interesting approach is the $f(T)$ gravity where $T$ is the torsion. Although it has no curvature, the space-time manifold has time evolution, and the dynamics has been caused by the Torsion only (Moller C. et al. 1961. 1978; Havashi K. et al.. 1967.1973.1977.1979; Wu P. et al. 2010.2011; Pellegrini C. et al. 1963; Yerzhanov K.K. et al. 2010; Chen S. H. et al. 2011; Bengochea G. R. et al. 2011; Li B. et al. 2011; Wei H. et al. 2011). Since its equations of motions are lower order, working with it is easier, also the matter comes from a non-minimally action and curvature. This model can be explained as the trace of the specified energy-momentum tensor, the common Ricci scalar, and the Lagrangian $f(R, T)$ Harko T. et al. (2011) and (Momeni D. et al. 2009, paper II). If we limit to the scalar field theories, there are many options to have an accelerated universe as quintessence (Zlatev I. et al. 
1999; Steinhardt P. J. et al. 1999) and (Amendola L. 2000, paper I), quintom models (Feng B. et al., 2006; Zhao W. 2006; Noiiri \& Odintsov 2006a, b), K-essense 1999,2000,2001) and other combined models. There is no limitation for the usage of the fermions in the matter sector of the theory as a matter source. There are some works on spinor cosmology in the literature (Armendariz-Picon C. et al. 1999,2000,2001, last paper ), (Vakili B. et al. 2005, paper II) and Ribas M. O. et al. 2005; Chaicherdsakul K. 2007; Boehmer C. G. 2008). In this work, following an earlier work on the generalization of the spinor cosmology (Jamil M. et al. 2011, paper II), we have introduced a new model of spinor cosmology, in which the action contains both $R$ and the non minimally coupled spinor fields. We also have investigated both isotropic and anisotropic cosmological models in this framework. We derive the basic equations of motion and solve them for FRW and Bianchi type-I models numerically. Finally both classical and quantum models in this framework have been discussed and some analytical solutions for the quantum cosmology have been studied.

\section{FRW metric in f-essence}

In this section we would like to present the derivation of the equations of motion for FRW metric in the fessence.

Let us consider the following action of f-essence

$S=\int d^{4} x \sqrt{-g}[R+2 K(Y, \psi, \bar{\psi})]$,

where $R$ is the scalar curvature, $Y$ is the kinetic term for the fermionic field $\psi$ and $K$ is some function of its arguments. In the case of the FRW metric

$d s^{2}=-d t^{2}+a^{2}\left(d x^{2}+d y^{2}+d z^{2}\right)$,

$R$ and $Y$ have the form

$R=6\left(\frac{\ddot{a}}{a}+\frac{\dot{a}^{2}}{a^{2}}\right)$,

$Y=0.5 i\left(\bar{\psi} \gamma^{0} \dot{\psi}-\dot{\bar{\psi}} \gamma^{0} \psi\right)$

respectively. Substituting these expressions into (1) and integrating over the spatial dimensions, we are led to an effective Lagrangian in the mini-superspace $\{a, \psi, \bar{\psi}\}$

$L=-2\left(3 a \dot{a}^{2}-a^{3} K\right)$.
Variation of Lagrangian (5) with respect to $a$, yields the equation of motion of the scale factor

mendariz-Picon C. et al.

$2 a \ddot{a}+\dot{a}^{2}+a^{2} K=0$.

The variation of Lagrangian (5) with respect to $\bar{\psi}, \psi$ is the corresponding Euler-Lagrangian equations for the fermionic fields

$$
\begin{aligned}
& K_{Y} \gamma^{0} \dot{\psi}+1.5 \frac{\dot{a}}{a} K_{Y} \gamma^{0} \psi \\
& +0.5 \dot{K}_{Y} \gamma^{0} \psi-i K_{\bar{\psi}}=0, \\
& K_{Y} \dot{\bar{\psi}} \gamma^{0}+1.5 \frac{\dot{a}}{a} K_{Y} \bar{\psi} \gamma^{0} \\
& +0.5 \dot{K}_{Y} \bar{\psi} \gamma^{0}+i K_{\psi}=0 .
\end{aligned}
$$

Another equivalence form is

$$
\begin{aligned}
& 3 H K_{\dot{\psi}}+K_{\dot{\psi} \psi} \dot{\psi}+K_{\dot{\psi} \bar{\psi}} \dot{\bar{\psi}} \\
& +K_{\dot{\psi} \dot{\psi}} \ddot{\psi}+K_{\dot{\psi} \dot{\bar{\psi}}} \ddot{\bar{\psi}}+K_{\psi}=0, \\
& 3 H K_{\dot{\bar{\psi}}}+K_{\dot{\bar{\psi}} \psi} \dot{\psi}+K_{\dot{\bar{\psi}} \bar{\psi}} \dot{\bar{\psi}} \\
& +K_{\dot{\bar{\psi}} \dot{\psi}} \ddot{\psi}+K_{\dot{\bar{\psi}} \dot{\bar{\psi}}} \ddot{\bar{\psi}}+K_{\bar{\psi}}=0 .
\end{aligned}
$$

Also the zero-energy condition is given by

$$
L_{\dot{a}} \dot{a}+L_{\dot{\psi}} \dot{\psi}+L_{\dot{\bar{\psi}}} \dot{\bar{\psi}}-L=0,
$$

which yields the constraint

$-3 a^{-2} \dot{a}^{2}+Y K_{Y}-K=0$.

Collecting all equations and rewriting using the Hubble parameter $H=(\ln a)_{t}$, we obtain a system of equations of f-essence (for the FRW metric case):

$$
\begin{aligned}
& 3 H^{2}-\rho=0, \\
& 2 \dot{H}+3 H^{2}+p=0, \\
& 3 H K_{\dot{\psi}}+K_{\dot{\psi} \psi} \dot{\psi}+K_{\dot{\psi} \bar{\psi}} \dot{\bar{\psi}} \\
& +K_{\dot{\psi} \dot{\psi}} \ddot{\psi}+K_{\dot{\psi} \dot{\bar{\psi}}} \ddot{\bar{\psi}}+K_{\psi}=0, \\
& 3 H K_{\dot{\bar{\psi}}}+K_{\dot{\bar{\psi}} \psi} \dot{\psi}+K_{\dot{\bar{\psi}} \bar{\psi}} \dot{\bar{\psi}} \\
& +K_{\dot{\bar{\psi}} \dot{\psi}} \ddot{\psi}+K_{\dot{\bar{\psi}} \dot{\bar{\psi}}} \ddot{\bar{\psi}}+K_{\bar{\psi}}=0, \\
& \dot{\rho}+3 H(\rho+p)=0 .
\end{aligned}
$$

Here

$\rho=Y K_{Y}-K, \quad p=K$,

are the energy density and the pressure of f-essence. It is clear that these expressions for the energy density and the pressure represent the components of the energymomentum tensor of f-essence as:

$T_{00}=Y K_{Y}-K, \quad T_{11}=T_{22}=T_{33}=-K$. 
3 FRW model with $K(Y, \psi, \bar{\psi})=Y-V(\bar{\psi} \psi)$

We introduce a useful model which is more applicable and more suitable for exact solutions. This model is described by

$K(Y, \psi, \bar{\psi})=Y-V(\bar{\psi} \psi)$.

To obtain the field equations, we substitute this form in Eqs. (13- 17). Another simple method is re-deriving these equations using the action directly, therefore in each of these equivalence methods we have the following equations of motion for Dirac fields:

$$
\begin{array}{r}
3 H\left(\frac{1}{2} i \bar{\psi} \gamma^{0}\right)+\frac{1}{2} i \gamma^{0} \dot{\bar{\psi}}-\frac{1}{2} i \dot{\bar{\psi}} \gamma^{0}-V_{\psi}=0 \\
3 H\left(-\frac{1}{2} i \gamma^{0} \psi\right)=V_{\bar{\psi}}
\end{array}
$$

FRW equations in this case are

$$
\begin{aligned}
& 2 \frac{\ddot{a}}{a}+\left(\frac{\dot{a}}{a}\right)^{2}+\frac{1}{2} i\left(\bar{\psi} \gamma^{0} \dot{\psi}-\dot{\bar{\psi}} \gamma^{0} \psi\right) \\
& -V(\bar{\psi} \psi)=0, \\
& 3 H^{2}=V(\bar{\psi} \psi) .
\end{aligned}
$$

The general potential is $V(\bar{\psi} \psi)=2 \bar{\psi} \psi$. For this special case, we have the next set of EOMs:

$$
\begin{array}{r}
3 H\left(\frac{1}{2} i \bar{\psi} \gamma^{0}\right)+\frac{1}{2} i \gamma^{0} \dot{\bar{\psi}}-\frac{1}{2} i \dot{\bar{\psi}} \gamma^{0}-2 \bar{\psi}=0 \\
-\frac{3}{2} i H \gamma^{0} \psi=2 \psi \\
2 \dot{H}+\frac{1}{2} i\left(\bar{\psi} \gamma^{0} \dot{\psi}-\dot{\bar{\psi}} \gamma^{0} \psi\right)=0 .
\end{array}
$$

Now we take the Dirac 2-spinor as $\bar{\psi}=\left(\psi_{1}, \psi_{2}\right)^{\dagger} \gamma^{0}$, the equation for spinor reads as

$\dot{\psi}+\frac{3}{2} H \psi+2 i \gamma^{0} \psi=0$

Thus we must solve the next system of ODEs:

$\frac{d \log \psi_{a}}{d t}=\frac{3}{2} H \psi_{a} \pm 2, a=\{1,2\}=\{+,-\}$,

which posses the following solution

$\psi^{T}=\left(\psi_{1}(0) a(t)^{3 / 2} e^{2 t}, \psi_{2}(0) a(t)^{3 / 2} e^{-2 t}\right)$.

Using this form of the 2-spinor we can obtain the scale factor from the following equation

$\ddot{y}+i e^{3 y}\left\{|\beta|^{2} e^{-4 t}-|\alpha|^{2} e^{4 t}\right\}$,

here $y \equiv \log (a(t)),\{\alpha, \beta\} \equiv\left\{\psi_{1}(0), \psi_{2}(0)\right\}$. There is no simple analytic solution for $y(t)$. But if we take $\alpha=\beta=\frac{1}{\sqrt{i}}$, then we can solve it numerically. FIG.1 shows the time evolution of $y(t)$ for some initial values.

\section{Bianchi type I cosmology of f-essence}

The action of f-essence reads as

$S=\int d^{4} x \sqrt{-g}[R+2 K(Y, \psi, \bar{\psi})]$

where $K$ is some function of its arguments, $\psi=$ $\left(\psi_{1}, \psi_{2}, \psi_{3}, \psi_{4}\right)^{T}$ is a fermionic function and $\bar{\psi}=\psi^{+} \gamma^{0}$ is its adjoint function. Here

$Y=0.5 i\left[\bar{\psi} \Gamma^{\mu} D_{\mu} \psi-\left(D_{\mu} \bar{\psi}\right) \Gamma^{\mu} \psi\right]$

is the canonical kinetic term for the fermionic field and $D_{\mu}$ is covariant derivative

$D_{\mu} \psi=\partial_{\mu} \psi+\Omega_{\mu} \psi, \quad D_{\mu} \bar{\psi}=\partial_{\mu} \bar{\psi}-\bar{\psi} \Omega_{\mu}$.

Here $\Omega_{\mu}$ are spin connections, $\Gamma^{\mu}$ are the Dirac matrices associated with the space-time metric satisfying the Clifford algebra

$\left\{\Gamma^{\mu}, \Gamma^{\nu}\right\}=2 g^{\mu \nu}$.

The $\Gamma^{\mu}$ are related to the flat Dirac matrices, $\gamma^{a}$, through the tetrads $e_{\mu}^{a}$ as

$\Gamma^{\mu}=e_{a}^{\mu} \gamma^{a}, \quad \Gamma_{\mu}=e_{\mu}^{a} \gamma_{a}$.

At the same time, the spin connections $\Omega_{\mu}$ satisfy the relation

$\Omega_{\mu}=0.25 g_{n u \lambda}\left(\partial_{\mu} e_{a}^{\lambda}+\Gamma_{\sigma \mu}^{\lambda} e_{a}^{\sigma}\right) \gamma^{\nu} \gamma^{a}$.

The tetrads can be easily obtained from their definition, that is

$g_{\mu \nu}=e_{\mu}^{a} e_{\nu}^{b} \eta_{a b}$

Let us now consider the Bianchi type I universe filled with f-essence. These models for the special simple spinors have been discussed previously Saha B. et al. 2004). The metric is given by

$d s^{2}=-N^{2}(t) d t^{2}+a^{2}(t) d x^{2}+b^{2}(t) d y^{2}+c^{2}(t) d z^{2}$,

where $a(t), b(t), c(t)$ are scale factors in the $x, y, z$ directions respectively and $N(t)$ is the lapse function. The corresponding scalar curvature takes the form

$$
\begin{aligned}
R= & \frac{2}{N^{2}}\left(\frac{\ddot{a}}{a}+\frac{\ddot{b}}{b}+\frac{\ddot{c}}{c}+\frac{\dot{a} \dot{b}}{a b}\right. \\
& \left.+\frac{\dot{a} \dot{c}}{a c}+\frac{\dot{b} \dot{c}}{b c}-\frac{\dot{a} \dot{N}}{a N}-\frac{\dot{b} \dot{N}}{b N}-\frac{\dot{c} \dot{N}}{c N}\right),
\end{aligned}
$$

where a dot represents differentiation with respect to $t$. For the metric (39) the tetrads take the form

$$
\begin{aligned}
& e_{\mu}^{a}=\operatorname{diag}(N, a, b, c), \\
& \quad e_{a}^{\mu}=\operatorname{diag}(1 / N, 1 / a, 1 / b, 1 / c) .
\end{aligned}
$$


These formulas yield

$$
\begin{aligned}
& \Omega_{0}=0, \quad \Omega_{1}=-\frac{\dot{a}}{2 N} \gamma^{0} \gamma^{1}, \quad \Omega_{2}=-\frac{\dot{b}}{2 N} \gamma^{0} \gamma^{2} \\
& \Omega_{3}=-\frac{\dot{c}}{2 N} \gamma^{0} \gamma^{3}
\end{aligned}
$$

where $\gamma^{0}$ and $\gamma^{i}$ are the Dirac matrices in Minkowski spacetime and we have adopted the following representation

$\gamma^{0}=\left(\begin{array}{cc}-i & 0 \\ 0 & i\end{array}\right), \gamma^{i}=\left(\begin{array}{cc}0 & \sigma^{i} \\ \sigma^{i} & 0\end{array}\right)$

Substituting (40) and (42) in (43) and integrating over the spatial dimensions, we are led to an effective Lagrangian in the mini-superspace $\{N, a, b, c, \psi, \bar{\psi}\}$

$L=-2\left[\frac{1}{N}(\dot{a} \dot{b} c+\dot{a} b \dot{c}+a \dot{b} \dot{c})-N a b c K(Y, \psi, \bar{\psi})\right]$

where

$L_{f}=2 N a b c K(Y, \psi, \bar{\psi}), \quad Y=\frac{1}{2 N}\left(\bar{\psi} \gamma^{0} \dot{\psi}-\dot{\bar{\psi}} \gamma^{0} \psi\right)$

The preliminary set-up for writing the equations of motion is now complete.

Variation of Lagrangian (44) with respect to $N, a, b, c, \psi$ and $\psi$ yields the equations of motion of the gravitational and the fermions fields as:

$$
\begin{aligned}
& \frac{\dot{a} \dot{b}}{a b}+\frac{\dot{a} \dot{c}}{a c}+\frac{\dot{b} \dot{c}}{b c} \\
& -N^{2}\left(Y K_{Y}-K\right)=0, \\
& \frac{\ddot{b}}{b}+\frac{\ddot{c}}{c}+\frac{\dot{b} \dot{c}}{b c} \\
& -\frac{\dot{N}}{N}\left(\frac{\dot{b}}{b}+\frac{\dot{c}}{c}\right)+N^{2} K=0, \\
& \frac{\ddot{a}}{a}+\frac{\ddot{c}}{c}+\frac{\dot{a} \dot{c}}{a c} \\
& -\frac{\dot{N}}{N}\left(\frac{\dot{a}}{a}+\frac{\dot{c}}{c}\right)+N^{2} K=0, \\
& \frac{\ddot{b}}{b}+\frac{\ddot{a}}{a}+\frac{\dot{b} \dot{a}}{b a} \\
& -\frac{\dot{N}}{N}\left(\frac{\dot{b}}{b}+\frac{\dot{a}}{a}\right)+N^{2} K=0, \\
& K_{Y} \dot{\psi}+0.5\left[(\ln (a b c))_{t} K_{Y}+\dot{K}_{Y}\right] \psi \\
& +N \gamma^{0} K_{\bar{\psi}}=0, \\
& K_{Y} \dot{\bar{\psi}}+0.5\left[(\ln (a b c))_{t} K_{Y}+\dot{K}_{Y}\right] \bar{\psi} \\
& -N K_{\psi} \gamma^{0}=0, \\
& \dot{\rho}+3 H(\rho+p)=0,
\end{aligned}
$$

where energy density and pressure take the form

$\rho=N^{2}\left(Y K_{Y}-K\right), \quad p=N^{2} K$

The vacuum solutions of the above system i.e. $\psi=0$ is the generalized Kasner solution in which space is homogeneous and has Euclidean metric depending on time according to the Kasner metric (Kasner, Edward 1921). This solution possessing a Belinsky-KhalatnikovLifshitz (BKL) singularity (Belinsky V.A. et al. 1969; Lifshitz E. M. et al. 1960), which is a model of dynamic evolution of the Universe near the initial singularity $t=0$ and described by an anisotropic homogeneous and chaotic solution to the Einstein's field equations of gravitation. The Mixmaster universe exhibits similar properties as the Kasner solution.

Some properties of g-essence were studied in (Kulnazarov I. et al. 2011; Razina O.V. et al. 2011). Model (1) admits two important reductions: $k$-essence and f-essence.

\section{Bianchi type I cosmologies with $\boldsymbol{K}(\boldsymbol{Y}, \boldsymbol{\psi}, \bar{\psi})=\boldsymbol{Y}-\boldsymbol{V}(\bar{\psi} \psi)$}

In this section we examine the Bianchi type I cosmology for $K(Y, \psi, \bar{\psi})=Y-V(\bar{\psi} \psi)$. In this case we have

$$
\begin{aligned}
& \frac{\dot{a} \dot{b}}{a b}+\frac{\dot{a} \dot{c}}{a c}+\frac{\dot{b} \dot{c}}{b c} \\
& -N^{2} V(\bar{\psi} \psi)=0, \\
& \frac{\ddot{b}}{b}+\frac{\ddot{c}}{c}+\frac{\dot{b} \dot{c}}{b c} \\
& -\frac{\dot{N}}{N}\left(\frac{\dot{b}}{b}+\frac{\dot{c}}{c}\right)+N^{2} K=0, \\
& \frac{\ddot{a}}{\bar{a}}+\frac{\ddot{c}}{c}+\frac{\dot{a} \dot{c}}{a c}-\frac{\dot{N}}{N}\left(\frac{\dot{a}}{a}+\frac{\dot{c}}{c}\right) \\
& +N^{2} K=0, \\
& \ddot{b}+\frac{\ddot{a}}{a}+\frac{\dot{b} \dot{a}}{b a}-\frac{\dot{N}}{N}\left(\frac{\dot{b}}{b}+\frac{\dot{a}}{a}\right) \\
& +N^{2} K=0, \\
& \dot{\psi}+0.5(\ln (a b c))_{t} \psi \\
& -N \gamma^{0} V_{\bar{\psi}}=0, \\
& \dot{\bar{\psi}}+0.5(\ln (a b c))_{t} \bar{\psi} \\
& +N V_{\psi} \gamma^{0}=0, \\
& \dot{\rho}+3 H(\rho+p)=0 .
\end{aligned}
$$


We take $V(\bar{\psi} \psi)=2 \bar{\psi} \psi$. Thus we obtain

$$
\begin{aligned}
& \frac{\dot{a} \dot{b}}{a b}+\frac{\dot{a} \dot{c}}{a c}+\frac{\dot{b} \dot{c}}{b c} \\
& -2 N^{2} \bar{\psi} \psi=0 \\
& \frac{\ddot{b}}{\bar{b}}+\frac{\ddot{c}}{c}+\frac{\dot{b} \dot{c}}{b c}-\frac{\dot{N}}{N}\left(\frac{\dot{b}}{b}+\frac{\dot{c}}{c}\right) \\
& +N^{2}\left(\frac{1}{2 N}\left(\bar{\psi} \gamma^{0} \dot{\psi}-\dot{\bar{\psi}} \gamma^{0} \psi\right)-2 \bar{\psi} \psi\right)=0, \\
& \frac{\ddot{a}}{\bar{a}}+\frac{\ddot{c}}{c}+\frac{\dot{a} \dot{c}}{a c}-\frac{\dot{N}}{N}\left(\frac{\dot{a}}{\bar{a}}+\frac{\dot{c}}{c}\right) \\
& +N^{2}\left(\frac{1}{2 N}\left(\bar{\psi} \gamma^{0} \dot{\psi}-\dot{\bar{\psi}} \gamma^{0} \psi\right)-2 \bar{\psi} \psi\right)=0 \\
& \ddot{b}+\ddot{a} \\
& \bar{b}+\frac{\dot{b} \dot{a}}{\bar{b}}-\frac{\dot{N}}{N}\left(\frac{\dot{b}}{\bar{b}}+\frac{\dot{a}}{a}\right) \\
& +N^{2}\left(\frac{1}{2 N}\left(\bar{\psi} \gamma^{0} \dot{\psi}-\dot{\bar{\psi}} \gamma^{0} \psi\right)-2 \bar{\psi} \psi\right)=0 \\
& \dot{\psi}+0.5(\ln (a b c))_{t} \psi-2 N \gamma^{0} \psi=0 \\
& \dot{\bar{\psi}}+0.5(\ln (a b c))_{t} \bar{\psi}+2 N \bar{\psi} \gamma^{0}=0, \\
& \dot{\rho}+3 H(\rho+p)=0 .
\end{aligned}
$$

We solved these equations numerically for a set of initial conditions imposed on the set of the functions $\left\{a(t), b(t), c(t),|\psi(t)|^{2}\right\}$. We set $N(t)=1$ without loss of generality, since the metric is a projectable metric, i.e. we can define a new time coordinate $t^{\prime}=\int N(t) d t$. The gauge $N(t)=1$ chosen in classical cosmological models, and called the cosmic time gauge. Another gauge fixing leads to $N_{i}=0$, here $N_{i}$ is the shift vector.

The numerical solutions is shown in the FIG.2. As we observe, the functions $\{a(t), b(t), c(t)\}$ are monotonically increasing functions of time, but the density function $|\psi(t)|^{2}$ is a decreasing function of $t$.

\section{Quantization of f-essence}

For quantization of the model as described in (32) we adopt the method proposed by Misner Misner C. W. 1969). The first step is writing the general Hamiltonian suitable for describing the quantum evolution of the system. For a typical model (32), with an unknown form of the function $K$, it is not possible to write such Hamiltonian. But if we restrict to the case $K(Y, \psi, \bar{\psi})=$ $Y-V(\bar{\psi} \psi)$, the problem at hand becomes tractable. This is a special case of the form discussed previously in (Vakili B. et al. 2005, paper I). First we introduce a set of the metric functions

$$
\begin{gathered}
\chi=\frac{1}{2 \sqrt{3}} \log \left(\frac{a}{b}\right), \\
\rho=\log (\sqrt{c \sqrt{a b}}), \\
\sigma=\frac{1}{2} \log \left(\frac{\sqrt{a b}}{c}\right) .
\end{gathered}
$$

It is easy to show that the Hamiltonian for model $K(Y, \psi, \bar{\psi})=Y-V(\bar{\psi} \psi)$ is

$$
H=\frac{e^{-3 \rho}}{12}\left(p_{\rho}^{2}-p_{\sigma}^{2}-p_{\chi}^{2}\right)+e^{3 \rho}[V(\bar{\psi} \psi)-\Upsilon]=0,
$$

where $\Upsilon$ is the Lagrange multiplier of the system. The set of the corresponding conjugate momentums of the new set of configurational coordinates $\{\rho, \sigma, \chi\}$ is $\left\{p_{\rho}, p_{\sigma}, p_{\chi}\right\}$ which satisfy the commutation brackets. With a specified form of the interaction $V(\bar{\psi} \psi)$, we can obtain the classical solutions described in (Vakili B. et al. 2005, paper I). Now from (71), we get the wave function directly from the WD equation (DeWitt B. S. 1967), and with the usual replacements $p_{i} \rightarrow-i \frac{\partial}{\partial x_{i}}$, the Wheeler-De Witt equation is

$$
\left[\frac{1}{12}\left(-\partial_{\rho}^{2}+\partial_{\sigma}^{2}+\partial_{\chi}^{2}\right)+e^{6 \rho}\left(-e^{-3 \rho}-\Upsilon\right)\right] \Psi(\rho, \sigma, \chi)=0
$$

We write the wave function as $\Psi(\rho, \sigma, \chi)=e^{i\left(k_{\sigma} \sigma+k_{\chi} \chi\right)} \Gamma(\rho)$ where

$$
-\partial_{\rho}^{2} \Gamma(\rho)+\left[-k_{\sigma}^{2}-k_{\chi}^{2}+12 e^{6 \rho}\left(-e^{-3 \rho}-\Upsilon\right)\right] \Gamma(\rho)=0 .
$$

The general solution for (73) is

$$
\begin{array}{r}
\Gamma(\rho)=\mathrm{e}^{-3 / 2 \rho}\left[C_{1} \mathbf{M}\left(\frac{-1 / 3 i \sqrt{3}}{\sqrt{\Upsilon}}, 1 / 3 i k, 4 / 3 i \sqrt{3} \sqrt{\Upsilon} \mathrm{e}^{3 \rho}\right)\right. \\
\left.+C_{2} \mathbf{W}\left(\frac{-1 / 3 i \sqrt{3}}{\sqrt{\Upsilon}}, 1 / 3 i k, 4 / 3 i \sqrt{3} \sqrt{\Upsilon} \mathrm{e}^{3 \rho}\right)\right] .
\end{array}
$$

Here $M$ and $W$ are Whittaker $M$ and Whittaker $W$ functions respectively while $k=\sqrt{k_{\sigma}^{2}+k_{\chi}^{2}}$. Thus the total wave function is

$$
\begin{aligned}
\Psi(\rho, \sigma, \chi)= & \sum_{k_{\sigma}, k_{\chi}} e^{i\left(k_{\sigma} \sigma+k_{\chi} \chi\right)} \mathrm{e}^{-3 / 2 \rho} \\
& \times\left[a_{k} \mathbf{M}\left(\frac{-1 / 3 i \sqrt{3}}{\sqrt{\Upsilon}}, 1 / 3 i k, 4 / 3 i \sqrt{3} \sqrt{\Upsilon} \mathrm{e}^{3 \rho}\right)\right. \\
& \left.+b_{k} \mathbf{W}\left(\frac{-1 / 3 i \sqrt{3}}{\sqrt{\Upsilon}}, 1 / 3 i k, 4 / 3 i \sqrt{3} \sqrt{\Upsilon} \mathrm{e}^{3 \rho}\right)\right] .
\end{aligned}
$$

For normalization we set $b_{k}=0$ and truncate the series for convergence. Thus the solution is written in final 
form as

$$
\begin{aligned}
\Psi(\rho, \sigma, \chi)= & \sum_{k_{\sigma}, k_{\chi}} e^{i\left(k_{\sigma} \sigma+k_{\chi} \chi\right)} \mathrm{e}^{-3 / 2 \rho} a_{k} \\
& \times \mathbf{M}\left(\frac{-1 / 3 i \sqrt{3}}{\sqrt{\Upsilon}}, 1 / 3 i k, 4 / 3 i \sqrt{3} \sqrt{\Upsilon} \mathrm{e}^{3 \rho}\right)
\end{aligned}
$$

The Fourier-Whittaker coefficients $a_{k}$ can be obtained from the initial wave function $\Psi(0, \sigma, \chi)$. For Gaussian wave packet we can obtain the following result for the Fourier amplitude as follow:

$a_{k}=\frac{e^{-\frac{k^{2}}{2}}}{2 \pi}$.

In Eq. (77) we use the approximation $\Upsilon \ll 1$.

\section{Conclusion}

In conclusion, we derived the equations of motion of f-essence for FRW and Bianchi type I metrics. It is shown that if the Lagrangian of fermionic fields $K$ has the usual Dirac form than the corresponding results coincide with the standard Einstein-Dirac theory. We have investigated both classical and quantum aspects of this model.

\section{Acknowledgments}

R. Myrzakulov would like to thank D. Singleton and Department of Physics, California State University Fresno for their hospitality during his one year visit (October, 2010 - October, 2011).

\section{Appendix}

As a double check, one can obtain the above field equations from the Einstein and Dirac equations given by:

$$
\begin{aligned}
R_{\mu \nu}-\frac{1}{2} R g_{\mu \nu} & =T_{\mu \nu} \\
\Gamma^{\mu} D_{\mu} \psi+K_{\bar{\psi}} & =0 \\
D_{\mu} \bar{\psi} \Gamma^{\mu}+K_{\psi} & =0 \\
\dot{\rho}+3 H(\rho+p) & =0 .
\end{aligned}
$$

For a homogeneous fermionic field $\psi(t)$, equations (A2) and (A3) are equivalent to (A1) and (A4) respectively. On the other hand, the non-vanishing components of the Einstein tensor for the metric (39) are:

$$
\begin{aligned}
G_{00} & =\frac{\dot{a} \dot{b}}{a b}+\frac{\dot{a} \dot{c}}{a c}+\frac{\dot{b} \dot{c}}{b c} \\
G_{11} & =-\frac{a^{2}}{N^{2}}\left[\frac{\ddot{b}}{b}+\frac{\ddot{c}}{c}+\frac{\dot{b} \dot{c}}{b c}-\frac{\dot{N}}{N}\left(\frac{\dot{b}}{b}+\frac{\dot{c}}{c}\right)\right] \\
G_{22} & =-\frac{b^{2}}{N^{2}}\left[\frac{\ddot{a}}{a}+\frac{\ddot{c}}{c}+\frac{\dot{a} \dot{c}}{a c}-\frac{\dot{N}}{N}\left(\frac{\dot{a}}{a}+\frac{\dot{c}}{c}\right)\right] \\
G_{33} & =-\frac{c^{2}}{N^{2}}\left[\frac{\ddot{b}}{b}+\frac{\ddot{a}}{a}+\frac{\dot{b} \dot{a}}{b a}-\frac{\dot{N}}{N}\left(\frac{\dot{b}}{b}+\frac{\dot{a}}{a}\right)\right]
\end{aligned}
$$

The components of the energy-momentum tensor for the fermionic field as the matter source can be obtained from the standard definition as:

$T_{\mu \nu}=2 \frac{\partial L_{f}}{\partial g^{\mu \nu}}-g_{\mu \nu} L_{f}$,

yielding

$$
\begin{gathered}
T_{00}=-2 N^{2}\left(Y K_{Y}-K\right), \quad T_{11}=-2 a^{2} K, \quad T_{22}=-2 b^{2} K, \\
T_{33}=-2 c^{2} K, \quad T_{i j}=T_{0 i}=0 .
\end{gathered}
$$

Substituting these results into Einstein equations (A1), yields the same equations as (46) -(52). In the case of the FRW metric (3), the equations corresponding to the action (1) can be obtained as:

$$
\begin{aligned}
3 H^{2}-\rho & =0, \\
2 \dot{H}+3 H^{2}+p & =0, \\
K_{Y} \dot{\psi}+0.5\left(3 H K_{Y}+\dot{K}_{Y}\right) \psi-i \gamma^{0} K_{\bar{\psi}} & =0, \\
K_{Y} \dot{\bar{\psi}}+0.5\left(3 H K_{Y}+\dot{K}_{Y}\right) \bar{\psi}+i K_{\psi} \gamma^{0} & =0 \\
\dot{\rho}+3 H(\rho+p) & =0,
\end{aligned}
$$

where the kinetic terms, the energy density and the pressure take the forms

$Y=0.5 i\left(\bar{\psi} \gamma^{0} \dot{\psi}-\dot{\bar{\psi}} \gamma^{0} \psi\right)$

$\rho=K_{Y} Y-K, \quad p=K$. 


\section{References}

Luca Amendola , Phys.Rev. D62 (2000) 043511; L. Amendola, S. Tsujikawa, Dark Energy: Theory and Observations, Cambridge University Press, 2010.

Armendariz-Picon C., Damour T., Mukhanov V.F., (1999) Phys. Lett. B 458, 209; Armendariz-Picon C., Mukhanov V.F., Steinhardt P.J., (2001) Phys. Rev. D 63, 103510; Armendariz-Picon C., Mukhanov V.F., Steinhardt P.J., (2000) Phys. Rev. Lett. 85, 4438;

C. Armendariz-Picon and P. B. Greene, Gen. Rel. Grav. 35 (2003) 1637, (hep-th/0301129).

A. Azadi, D. Momeni, M. Nouri-Zonoz, Phys.Lett.B670:210$214,2008$.

V.A. Belinsky , I.M. Khalatnikov (1969). JETP 57: 2163.

Kazuharu Bamba, Sergei D. Odintsov, Lorenzo Sebastiani, Sergio Zerbini, Eur.Phys.J. C67 (2010) 295-310.

Gabriel R. Bengochea, Phys.Lett. B695 (2011) 405-411

Christian G. Boehmer, Phys.Rev.D77:123535,2008.

Kanokkuan Chaicherdsakul, Phys.Rev.D75:063522,2007.

Shih-Hung Chen, James B. Dent , Sourish Dutta, Emmanuel N. Saridakis, Phys.Rev. D83 (2011) 023508.

G. Cognola et al., Phys. Rev. D 77 (2008) 046009.

Copeland E. J., Sami M., Tsujikawa S., (2006) Int. J. Mod. Phys. D 15, 1753.

B. S. DeWitt (1967) Phys. Rev. 1601113.

Emilio Elizalde, Shin'ichi Nojiri, Sergei D. Odintsov, Phys.Rev.D70:043539,2004; E. Elizalde , R. Myrzakulov , V.V. Obukhov, D. Saez-Gomez, Class.Quant.Grav. 27 (2010) 095007; E. Elizalde et al., Phys. Rev. D 83 (2011) 086006.

Antonio De Felice, Jean-Marc Gerard, Teruaki Suyama, Phys.Rev. D82 (2010) 063526.

Bo Feng, Mingzhe Li , Yun-Song Piao , Xinmin Zhang , Phys.Lett. B634 (2006) 101-105.

Tiberiu Harko , Francisco S.N. Lobo , Shin'ichi Nojiri , Sergei D. Odintsov , Phys.Rev. D84 (2011) 024020.

K. Hayashi and T. Nakano, Prog. Theor. Phys. 38, 491 (1967); K. Hayashi, Nuovo Cimento A 16, 639 (1973); K. Hayashi, Phys. Lett. B 69, 441 (1977); K. Hayashi and T. Shirafuji, Phys. Rev. D 19: 3524-3553 (1979).

M. Jamil, Fazal M. Mahomed , D. Momeni, Phys.Lett.B702 (2011) 315-319; M. Jamil, Y. Myrzakulov, O. Razina, R. Myrzakulov, Astrophys Space Sci (2011) 336:315-325.

Kasner, Edward (1921) American Journal of Mathematics 43 (4): 217221.

I. Kulnazarov, K. Yerzhanov, O. Razina, Sh. Myrzakul, P. Tsyba, R. Myrzakulov, Eur. Phys. J. C, 71:1698 (2011).

Baojiu Li, Thomas P. Sotiriou, John D. Barrow , Phys.Rev. D83 (2011) 064035.

Evgeny M. Lifshitz,I.M. Khalatnikov (1960). JETP 39: 149; Evgeny M. Lifshitz, I.M. Khalatnikov (1960). JETP 39: 800.

C. W. Misner, Phys. Rev. 186, 1328 (1969); C. W. Misner, Phys. Rev. 186, 1319 (1969).

C. Moller, Mat. Fys. Skr. Dan. Vid. Selsk. 1 no. 10 (1961); C. Moller, K. Dan. Vidensk. Selsk. Mat. Fys. Skr. 89, No. 13 (1978).

D. Momeni, H. Gholizade, Int.J.Mod.Phys.D18:1719-1729,2009; D. Momeni, Mubasher Jamil, Ratbay Myrzakulov, arXiv:1107. $\$ 907$
S. Nojiri, S.D. Odintsov, Int. J. Geom. Meth. Mod. Phys. 4 (2007) 115; S. Nojiri, S.D. Odintsov, Phys.Rept.505:59144,2011;S. Nojiri, S.D. Odintsov, Phys. Rev. D 68 (2003) 123512 .

C. Pellegrini and J. Plebanski, Mat. Fys. Skr. Dan. Vid. Selsk. 2 no. 4 (1963).

Perlmutter S. , (1999) Astrophys. J. 517, 565.

Puxun Wu , Hong Wei Yu, Phys.Lett. B693 (2010) 415-420; Puxun Wu, Hong Wei Yu, Eur.Phys.J. C71 (2011) 1552.

O.V. Razina, Y.M. Myrzakulov, N.S. Serikbayev, Sh.R. Myrzakul, G.N. Nugmanova, R. Myrzakulov, Eur. Phys. J. Plus (2011) 126: 85.

M. O. Ribas, F. P. Devecchi, G. M. Kremer, Phys.Rev. D72 (2005) 123502.

A.G. Riess, (1998) Astron. J. 116, 1009.

Bijan Saha, Todor Boyadjiev, Phys.Rev. D69 (2004) 124010.

Paul J. Steinhardt, Li-Min Wang, Ivaylo Zlatev (, Phys.Rev. D59 (1999) 123504.

B. Vakili, H. R. Sepangi, JCAP09(2005)008;

B. Vakili, H.R. Sepangi Time reparameterization in Bianchi type I spinor cosmology, AnnalsPhys.323:548565,2008, arXiv:0709.2988.

Hao Wei, Xiao-Peng Ma, Hao-Yu Qi , Phys.Lett. B703 (2011) 74-80.

K.K. Yerzhanov, Sh.R. Myrzakul, I.I. Kulnazarov, R. Myrzakulov, arXiv:1006.3879 [gr-qc].

Wen Zhao, Phys.Rev. D73 (2006) 123509.

S. Nojiri, S. D. Odintsov, Gen.Rel.Grav.38:1285-1304,2006.

S. Nojiri, S. D. Odintsov, Gen.Rel.Grav.38:1285-1304,2006.

Ivaylo Zlatev, Li-Min Wang, Paul J. Steinhardt , Phys.Rev.Lett. 82 (1999) 896-899.

This manuscript was prepared with the AAS LATEX macros v5.2.



Numerical solution for $y(t)=\log (a(t))$. 


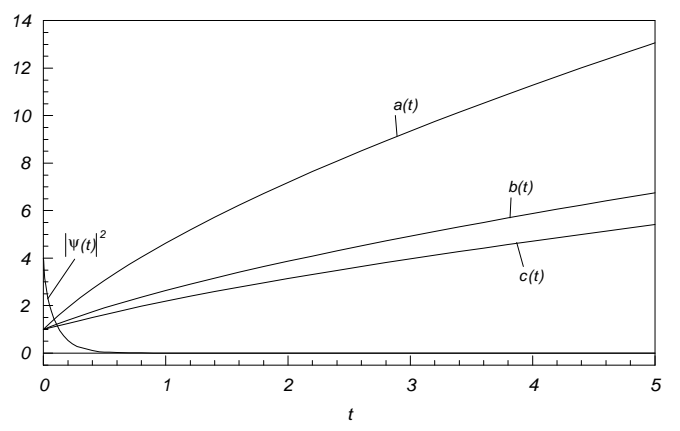

Fig. 2 Numerical solution for $\left\{a(t), b(t), c(t),|\psi(t)|^{2}\right\}$. 